\title{
Description des propriétés mécaniques de la Molasse aquitanienne de Lausanne
}

\author{
Mustafa Gencer ${ }^{1, \star}$, Jean-François Mathier ${ }^{2}$, Francis Noverraz ${ }^{1}$ et Olivier Français ${ }^{3}$ \\ ${ }^{1}$ Karakas \& Français SA, Lausanne, Suisse \\ ${ }^{2}$ Laboratoire de Mécanique des Roches de l'École Polytechnique Fédérale (EPFL), Lausanne, Suisse \\ ${ }^{3}$ Municipal de la Ville de Lausanne, Lausanne, Suisse
}

\begin{abstract}
Résumé - Le tunnel ferroviaire de la société TRIDEL SA à Lausanne (Suisse) a été entièrement réalisé dans la formation molassique caractéristique de la région. Le présent article propose une classification des différents types de roches constituant cette formation molassique (Molasse aquitanienne ou Molasse grise de Lausanne) sur la base des critères lithologiques établis selon les relevés des géologues. Les caractéristiques mécaniques propres à chaque type de roche ont été déterminées à partir des essais de résistance uniaxiale avec mesure du module d'élasticité, des essais triaxiaux et des essais brésiliens. Les corrélations entre les différentes propriétés mécaniques sont analysées en fonction des types de molasse sur plus de 350 échantillons prélevés lors de la réalisation du tunnel. Les corrélations obtenues ont été utilisées pour définir les paramètres des critères de rupture pour la roche intacte selon Hoek \& Brown et Mohr-Coulomb. Dans le cas particulier des marnes (roches argileuses tendres), les résultats des essais triaxiaux ont été analysés en appliquant les principes de l'état critique par analogie à la mécanique des sols pour définir les paramètres des lois de rupture.
\end{abstract}

Mots clés : Molasse aquitanienne / classification lithologique / essais en laboratoire / propriétés mécaniques / critères de rupture

\begin{abstract}
Description of the mechanical characteristics of the Aquitanien Molasse of Lausanne. The TRIDEL company railway tunnel in Lausanne (Switzerland) was built entirely within the characteristic Molassic Formation of the region. The following paper offers a classification of the various types of rock that make up this Molassic Formation (Aquitanian Molasse or grey Molasse of Lausanne) based on lithologic criteria established according to geological surveys. The mechanical characteristics for each type of rock were determined from uniaxial compression tests that provide Young's modulus, as well as from triaxial and Brazilian tests. The correlations between these various mechanical properties were analyzed as a function of the types of Molasse on more than 350 samples taken during the construction of the tunnel. The correlations thus obtained were used to define the failure criteria parameters for the intact rock according to Hoek and Brown and Mohr-Coulomb. In the particular case of Claystones (soft clayey rock), the triaxial tests results were analyzed by using principles of Critical State as in soil mechanics in order to determine the parameters of those failure criterions.
\end{abstract}

Keywords: Aquitanian Molasse / lithologic classification / laboratory tests / mechanical properties / failure criterion / the failure criteria

\section{Introduction}

La société TRIDEL SA constituée dans le cadre du projet de construction d'une usine d'incinération à Lausanne a décidé de réaliser un tunnel ferroviaire à voie unique pour acheminer les déchets incinérables par trains, avec une pente de près de

\footnotetext{
$\bar{\star}$ Auteur de correspondance :

mustafa.gencer@karakas-francais.ch
}

$5 \%$ depuis la gare de Sébeillon (altitude $420 \mathrm{~m} / \mathrm{mer}$ ) au bas de la ville jusqu'à l'usine située sur le plateau de la Sallaz (altitude $600 \mathrm{~m} / \mathrm{mer}$ environ). Ce tunnel d'une longueur totale de $4 \mathrm{~km}$ environ a été creusé, sur un tronçon de $3,5 \mathrm{~km}$, entièrement dans la Molasse aquitanienne à grande profondeur sous la ville durant les années 2005 et 2006.

La présente étude propose une description détaillée des différents faciès de roche constituant la formation molassique sur la base des critères lithologiques. En pratique, dans le cadre des études préliminaires, les faciès ont été groupés en 


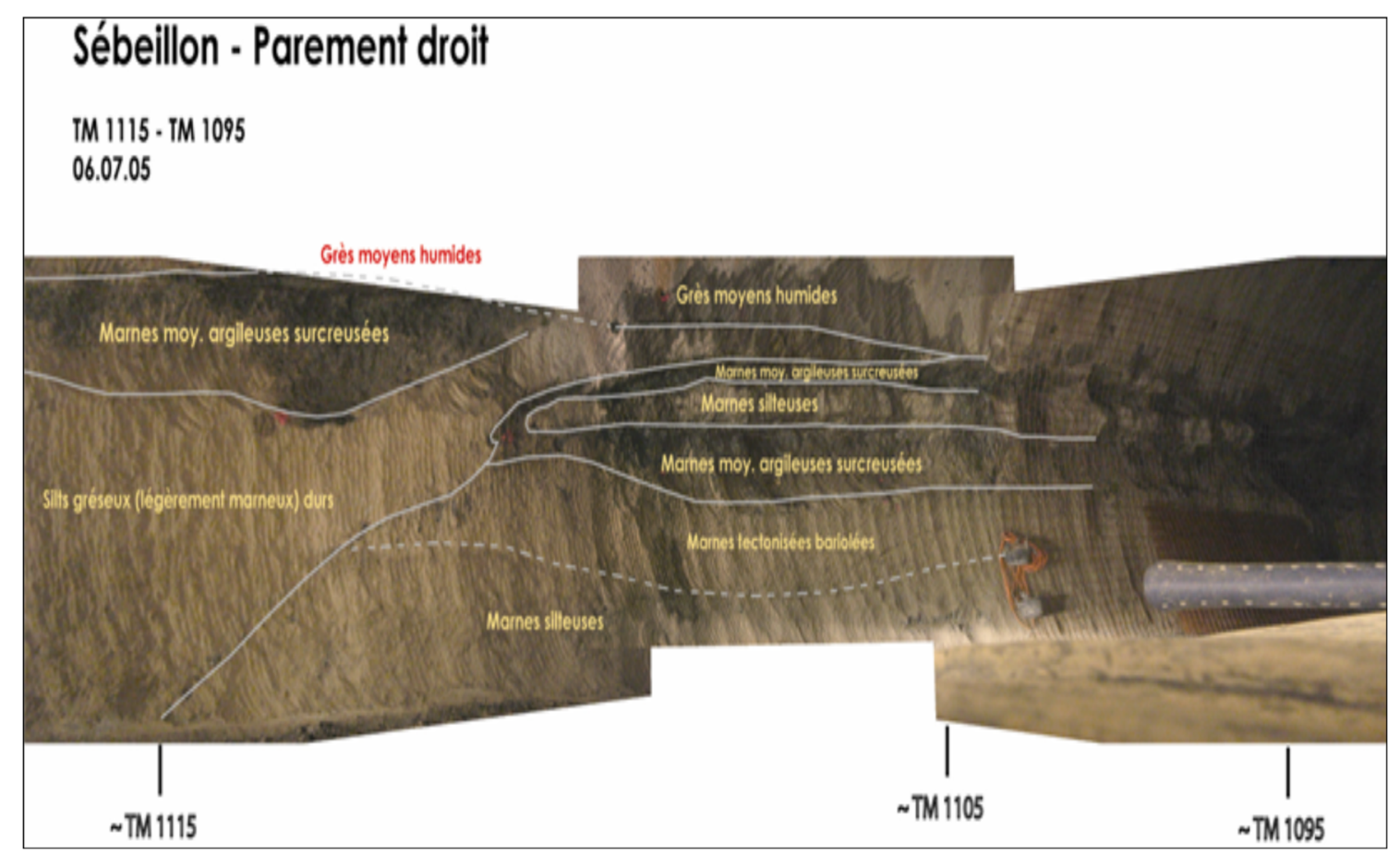

Photo 1. Faciès de molasse relevés lors de la creuse du tunnel.

Photo 1. Layers of Molasse observed during the excavation of the tunnel.

catégories de roche en fonction de leurs propriétés mécaniques (résistance, dureté, abrasivité) et de leur comportement prévisible (altérabilité, temps de tenue sans soutènement, gonflement, etc.) lors des travaux d'exécution de tunnel.

$\mathrm{Au}$ cours de la réalisation de ce tunnel, plus de 350 échantillons ont été prélevés pour être soumis à des essais de mécanique des roches au laboratoire de l'EPFL. La description lithologique de ces échantillons de Molasse a été effectuée par trois géologues lors de l'exécution. Les caractéristiques mécaniques propres à chaque type de roche ont été déterminées à partir des essais de compression simple avec mesure du module d'élasticité et essais brésiliens. Les corrélations entre ces différentes propriétés mécaniques sont analysées en fonction des types de Molasse.

Les résultats des essais triaxiaux réalisés exclusivement sur les échantillons de marne ont permis de définir les paramètres des critères de rupture pour ce type de Molasse selon Hoek \& Brown, Mohr-Coulomb et la théorie de l'état critique.

\section{Conditions géologiques générales}

Le soubassement rocheux de la ville de Lausanne est constitué dans sa presque totalité par la Molasse d'eau douce inférieure, d'âge aquitanien (Miocène inférieur), appelée aussi Molasse grise de Lausanne.

Cette formation rocheuse est constituée d'une alternance d'assises gréseuses à ciment calcaire, souvent épaisses de plusieurs mètres, voire de plus de $10 \mathrm{~m}$, et de couches diverses à composante marno-argileuse plus ou moins importante, incluant des grès marneux, des marno-grès, des marnes silto- gréseuses, des silts plus ou moins marneux, et enfin des marnes plus ou moins argileuses.

Ces différents faciès ne sont pas présents en couches continues, mais résultent le plus souvent de modes de dépôt très chenalisés ou lenticulaires, en particulier en ce qui concerne les grès, avec de très faibles continuités latérales, pour les couches les plus grossières surtout, et avec des transitions parfois brutales et imprévisibles d'un faciès à l'autre qui rendent la prévision difficile (Photo 1).

La plupart de ces faciès rocheux sont de dureté faible (les marnes) à modérée (y compris les gros horizons de grès plus ou moins grossiers). Seuls les grès fins silteux et les silts (non marneux) présentent des duretés plus élevées.

Les marnes renferment en proportions variables des argiles à forte capacité de gonflement en présence d'eau, de la famille des smectites (montmorillonites - alias bentonites - surtout), qui ont pour conséquence que ces niveaux rocheux résistent mal à l'altération météorique et aux changements de teneur en eau (dessiccation, humidification excessive provoquant le gonflement).

Le gonflement de la roche qui en résulte conduit à une perte de cohésion et à sa désagrégation, ainsi que, le cas échéant, à des poussées sur les structures de revêtement et de soutènement.

Du point de vue structural, le massif molassique est caractérisé sur tout le tracé par un pendage stratigraphique très faible, oscillant entre 0 et 5 à $10^{\circ}$. Des inclinaisons plus fortes, parfois observées de manière localisée, peuvent être dues à des processus liés à la pédogenèse ou à des redressements locaux des couches à proximité d'un accident tectonique. De même, une intense microfissuration observée dans certaines marnes, gênante pour le prélèvement 
d'échantillons pour les essais en laboratoire, est d'origine pédogénétique et non pas tectonique.

La morphologie du toit de la formation rocheuse molassique lausannoise est caractérisée par de profondes entailles consécutives à l'érosion fluviale. Certaines sont bien visibles et déterminent la géomorphologie de la ville, tels les vallons du Flon, du Petit Flon (la Louve), de la Vuachère; d'autres sillons d'érosion de l'époque glaciaire sont dissimulés par la couverture quaternaire morainique et glacio-lacustre et doivent être déterminés par des travaux de reconnaissance par forages.

Le tunnel ferroviaire de Tridel, relativement profond (entre 10 et $70 \mathrm{~m}$, avec plusieurs dizaines de mètres de couverture rocheuse dans le tunnel), a été réalisé entièrement dans le soubassement rocheux molassique.

Comme l'avaient mis en évidence les reconnaissances par forages, le massif rocheux a montré de nettes différences de composition d'une partie à l'autre du tracé : il s'est révélé plus gréseux dans la partie haute de la ville et plus riche en marnes et faciès intermédiaires marno-gréso-silteux dans la partie inférieure du tracé.

Ces variations régionales du mode de dépôt, de la composition des couches et de leur alternance nécessitent pour des raisons pratiques de grouper les faciès en catégories (couches types) sur certains tronçons en fonction de leur lithologie prédominante. Les considérations statistiques sur les propriétés mécaniques d'une catégorie idéalisée établie sur la base des essais effectués montrent souvent une dispersion importante des résultats d'essais, notamment dans le cas des marnes de la Molasse Grise de Lausanne rencontrées lors de l'exécution du tunnel TRIDEL.

\section{Description lithologique des types de molasse}

Les roches de la Molasse aquitanienne du Plateau sont composées en gros de trois éléments qui donnent naissance aux différents faciès de cette formation rocheuse, ces faciès alternant par couches stratigraphiques successives, souvent sans grande continuité latérale (dépôts chenalisés) :

- la fraction granulaire, composée de grains de quartz et de différents minéraux tels que feldspaths, minéraux ferromagnésiens, minéraux du métamorphisme. Plus la granulométrie est fine, plus la proportion de quartz est largement prédominante;

- la granulométrie de cette fraction granulaire va des sables moyens à grossiers (environ $2 \mathrm{~mm}$ ) aux limons (ou silts) les plus fins (entre 0,06 et $0,002 \mathrm{~mm}$ selon les normes de la mécanique des sols);

- la fraction argileuse (granulométrie inférieure à 0,002 mm selon les normes de la mécanique des sols), composée de différents phyllosilicates (argiles diverses, micas, chlorite). À noter que la notion granulométrique n'est pas vraiment applicable à ces minéraux feuilletés ;

- la fraction calcaire, qui compose le ciment non cristallisé de la roche et, en bien moindre proportion, des grains calcaires pour les faciès les plus grossièrement granulaires.

Ces trois fractions forment entre elles, en proportions variables et en répartitions granulométriques diverses, l'ensemble des faciès sédimentologiques de la formation molassique.

Considérés sur l'ensemble du tracé, les différents types de lithologie de la Molasse rencontrés lors de la creuse du tunnel TRIDEL peuvent être décrits de la manière suivante :

- grès grossiers : présents surtout à la base de certains gros niveaux gréseux, ils sont statistiquement très peu représentés. Ce sont des roches mal cimentées, peu dures, mécaniquement peu résistantes à l'écrasement, au cisaillement et à la traction, fortement abrasives (70 à $80 \%$ de silice, dont 40 à $50 \%$ due au quartz);

- grès moyens: beaucoup plus fréquents que les grès grossiers, souvent en continuité avec ceux-ci, parfois en niveaux épais, ils ne diffèrent de ces derniers que par une résistance mécanique un peu supérieure;

- grès fins : ce sont les grès les plus fréquents reconnus sur le tracé ; ils passent souvent en continuité soit aux grès plus grossiers, soit aux silts. Plus riches en calcaire et en l'occurrence mieux cimentés que les faciès plus grossiers, ils sont durs et plus résistants mécaniquement;

- silts, silts gréseux, silts marneux : ce sont les roches les plus dures rencontrées sur le tracé. Bien cimentées, avec une proportion variable mais souvent assez importante de ciment calcaire, ils peuvent être très durs, et presque toujours massifs, sans litage, en bancs pluridécimétriques à plurimétriques dans de rares cas.

Souvent en continuité avec les faciès voisins, ces faciès intermédiaires sont tantôt à composante gréseuse (silts-marnogréseux) tantôt à composante silteuse et à ciment marneux (silts marneux), ou marno-calcaire (silts marno-calcaires).

Malgré leur dureté, ce sont des roches peu résistantes à l'altération météorique dès qu'elles renferment une composante argileuse (silts marneux, silts gréso-marneux) :

- marno-grès : plus rares que les silts marneux sur le tracé, ce sont des roches peu dures, peu résistantes mécaniquement et très sensibles à l'altération météorique. Elles peuvent donner lieu à un faible gonflement;

- marnes gréseuses, marnes silteuses: fréquentes, presque toujours à composante silteuse, ces roches sont peu dures (marnes gréseuses) à assez dures (marnes silteuses) et présentent des propriétés semblables aux marno-grès. Elles se fusent à l'air libre mais ne sont sujettes au gonflement que de manière négligeable. Toutes ces roches de faciès intermédiaires sont aussi désignées par le terme local de macignos, et, par simplification abusive, de marno-grès ;

- marnes : fréquentes et parfois présentes en niveaux épais, ce sont des roches tendres, très peu résistantes mécaniquement, qui se fusent rapidement à l'air libre. Elles donnent lieu à un gonflement variable selon les composantes argileuses et leur proportion, et selon la proportion de carbonate (les marnes très calcaires sont très faiblement gonflantes);

- marnes argileuses (marnes grasses) : ce sont des marnes dont la composante argileuse représente $75 \%$ ou davantage de la roche totale. Très tendres, elles donnent lieu à un fort gonflement lors de la suppression des contraintes, surtout en présence d'eau. Elles sont peu représentées dans la Molasse aquitanienne de Lausanne. 


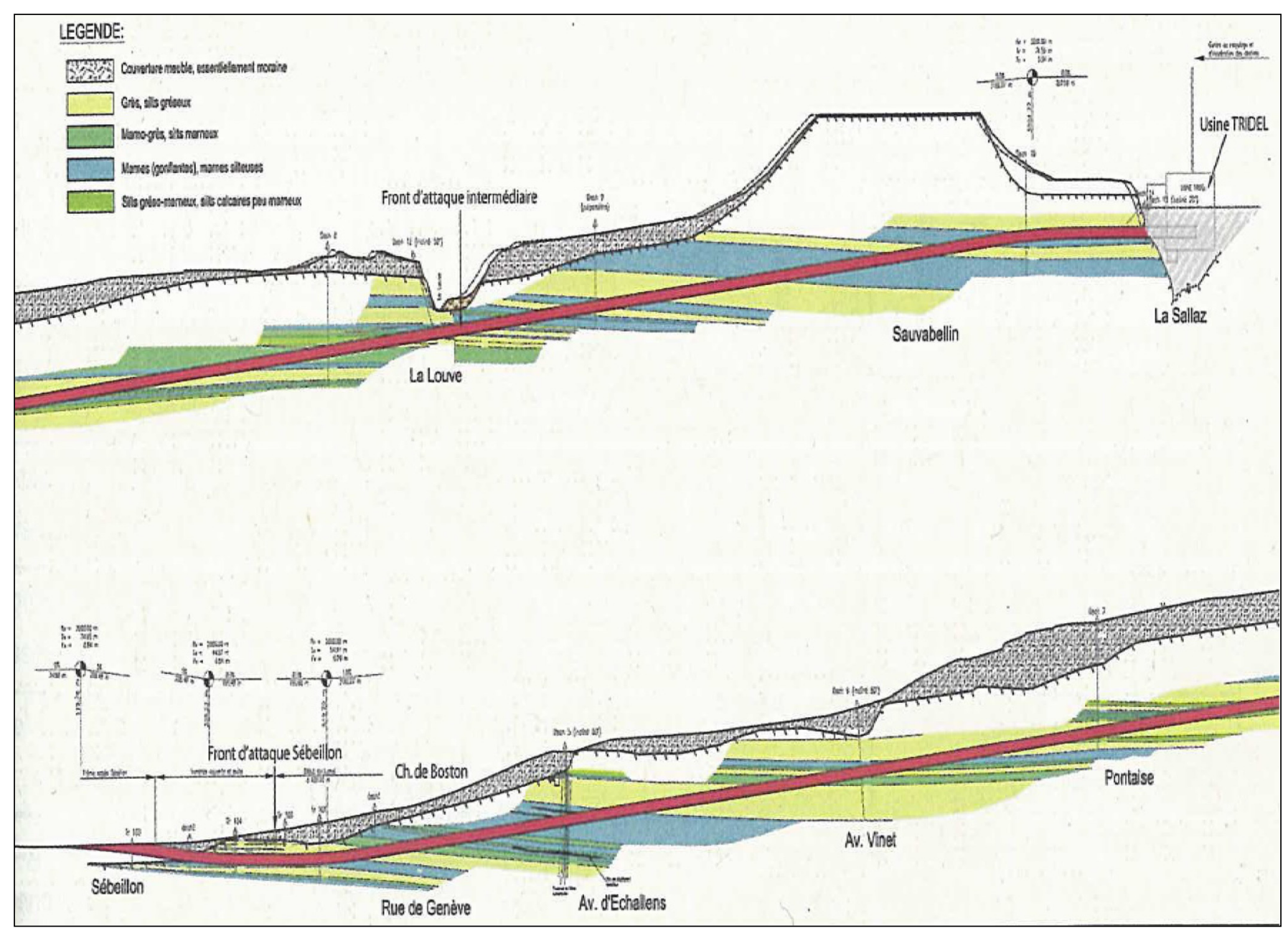

Fig. 1. Profil en long géologique prévisionnel en quatre catégories.

Fig. 1. Projected geological profile with four rock categories.

\section{Classification de la molasse selon les critères lithologiques et de dureté}

\subsection{Phase étude géologique}

Dans le cadre de l'étude géologique préliminaire de KARAKAS \& FRANÇAIS SA (2004) basée sur un nombre limité de forages, les différents faciès idéalisés de Molasse ont été représentés en quatre catégories principales selon leur composition lithologique :

- les grès (moyens et grossiers), et les grès silteux sans fraction argileuse;

- les silts gréseux-marneux et silts calcaires peu marneux qui constituent la composante la plus dure de la Molasse;

- les marnes et marnes argileuses, roches sans quartz, susceptibles de gonfler et les marnes silteuses sensibles à l'altération;

- les faciès intermédiaires, incluant les marno-grès, les silts plus ou moins marneux, les marnes silto-gréseuses, soit tous les faciès comportant une fraction argileuse plutôt faible et une fraction silto-gréseuse.

Le profil géologique en long du tunnel (Fig. 1) a été établi sur la base de cette classification en quatre catégories en tenant compte des critères sédimentologiques et stratigraphiques sur la base des corrélations pouvant être établies entre les forages.

Les échantillons prélevés dans des sondages ont été soumis essentiellement à des essais de résistance uniaxiale et brésiliens. Les résultats obtenus sur une vingtaine d'échantillons ont permis de regrouper les faciès en trois catégories caractéristiques selon leur dureté, comme indiqué dans le tableau 1.

Les valeurs de la première ligne pour chaque type de roche représentent la moyenne et l'écart-type. La troisième ligne correspond au coefficient de variation (rapport écart-type sur la moyenne). Les valeurs minimales et maximales obtenues sont indiquées à la deuxième ligne. En ce qui concerne les marnes, nous n'avons pu effectuer que deux essais car il est très difficile de préparer des éprouvettes d'essais dans cette roche très sensible.

$\mathrm{Au} \mathrm{vu} \mathrm{de} \mathrm{ces} \mathrm{résultats,} \mathrm{on} \mathrm{constate} \mathrm{que} \mathrm{les} \mathrm{faciès}$ intermédiaires caractérisés par le vocable macignos sont nettement plus durs que les grès. En revanche, la variabilité spatiale avec des coefficients de variation entre 60 et $75 \%$ de leur résistance selon les emplacements est beaucoup plus importante que celle des grès. 
Tableau 1. Propriétés mécaniques de la Molasse réunies en trois catégories.

Table 1. Mechanical properties for three Molasse formations.

\begin{tabular}{|c|c|c|c|c|}
\hline Type de molasse & $\begin{array}{l}\text { Résistance uniaxiale } \\
\sigma_{c}\left(\mathrm{MN} / \mathrm{m}^{2}\right)\end{array}$ & $\begin{array}{l}\text { Module d'élasticité } \\
E\left(\mathrm{MN} / \mathrm{m}^{2}\right)\end{array}$ & $\begin{array}{l}\text { Résistance à la traction } \\
\sigma_{t}\left(\mathrm{MN} / \mathrm{m}^{2}\right)\end{array}$ & $\begin{array}{l}\text { Résistance à l'écrasement } \\
\text { ponctuel } \mathrm{I}_{50}\left(\mathrm{MN} / \mathrm{m}^{2}\right)\end{array}$ \\
\hline \multirow[t]{2}{*}{ Grès moyen à grossier } & $17,5 \pm 5,8$ & $3856 \pm 1663$ & $0,65 \pm 0,36$ & $0,6 \pm 0,3$ \\
\hline & $33 \%$ & $43 \%$ & $55 \%$ & $50 \%$ \\
\hline \multirow{2}{*}{$\begin{array}{l}\text { Faciès gréso-marneux et silts } \\
\text { marneux (macignos) }\end{array}$} & $32,4 \pm 19,4$ & $5498 \pm 4572$ & $1,53 \pm 1$ & $1,6 \pm 1,2$ \\
\hline & $60 \%$ & $83 \%$ & $65 \%$ & $75 \%$ \\
\hline Marne & $2,8-5,7$ & $700-900$ & $0,2-0,59$ & $\begin{array}{l}0,3 \pm 0,1 \\
0,2-0,4\end{array}$ \\
\hline
\end{tabular}

\subsection{Phase exécution}

Lors du creusement du tunnel, des relevés géologiques ont été effectués régulièrement pour préciser les caractéristiques lithologiques des faciès rencontrés. En parallèle plus de 350 échantillons de Molasse ont été prélevés dans ces faciès par carottage au front d'excavation. Ils peuvent donc être considérés comme représentatifs de l'ensemble de la Molasse aquitanienne rencontrée dans le tunnel de TRIDEL. Ces carottes ont été décrites par trois géologues selon les critères lithologiques pour chaque faciès molassique. On distingue au total douze types de faciès définis par les géologues. Ces faciès ont été réunis en cinq classes sur la base d'une analyse statistique (tests d'hypothèse de comparaison de la différence des valeurs moyennes de résistance uniaxiale et à la traction pour chaque population de faciès) en fonction de leurs propriétés mécaniques déterminées à partir d'essais de compression simple et brésiliens en laboratoire.

Le tableau 2 résume les classifications établies selon les critères lithologiques et de dureté dans le cadre des études préliminaires et lors de l'exécution. En première colonne de ce tableau, on trouvera la description très sommaire utilisée parfois de manière abusive en pratique pour distinguer les catégories de Molasse.

Ce tableau donne le domaine de recouvrement de l'ensemble des faciès par les diverses catégorisations, notamment celles retenues lors des études géologiques préliminaires basées sur un nombre limité de forages. Il permet également d'établir les correspondances lithologiques entre les diverses descriptions géologiques utilisées en pratique pour qualifier la Molasse aquitanienne. La classification en 5 catégories a été reprise dans le cadre de la présente étude pour décrire les propriétés de cette molasse aux chapitres suivants.

\section{Influence des conditions géologiques sur le tunnel et les prévisions}

Le tunnel n'ayant jamais quitté le soubassement rocheux, les conditions géologiques rencontrées sont entièrement liées aux propriétés de la Molasse aquitanienne de Lausanne. Le tunnel a été creusé à partir de trois fronts d'attaque dont l'un est situé à Sébeillon au point bas du tracé. Les deux autres fronts ont été créés dans les sens montant et descendant à l'extrémité d'une galerie d'accès réalisée à cet effet depuis une zone forestière en dehors du site urbain, situé pratiquement à un tiers du tracé depuis l'usine TRIDEL.

Dans un premier temps, l'excavation a été effectuée à l'aide d'une machine équipée de marteau hydraulique (brise roche) avec des rendements qui se sont avérés faibles. Ensuite, le creusement a été poursuivi en recourant à une haveuse de puissance moyenne. Le tronçon supérieur du tunnel jusqu'à l'usine TRIDEL sur une longueur de $800 \mathrm{~m}$ environ a été réalisé par abattage à l'explosif.

Du point de vue de la dureté, aspect essentiel pour le projet en raison de l'utilisation d'une haveuse de taille et de puissance réduite, la roche rencontrée s'est avérée assez aisée à excaver, sauf en ce qui concerne certains secteurs à composante siltogréseuse assez calcaire : ce type de faciès présente des duretés assez élevées (souvent plus de $30 \mathrm{MN} / \mathrm{m}^{2}$ ) et une quasiabsence de litage stratigraphique, qui ont réduit le rendement de l'excavation.

Dans l'ensemble, le litage de la formation rocheuse s'est avéré peu accentué et n'a occasionné des problèmes de tenue en calotte que très localement, dans les faciès à composante marneuse.

La tectonisation de la roche s'est révélée peu importante; aucun accident tectonique important (chevauchement, décrochement, décollements ou zones de broyage) n'a été rencontré, pas même au passage sous le vallon de la Louve. Seules des fractures généralement subverticales et de rares failles ont été rencontrées; elles ont provoqué quelques problèmes très localisés de tenue des piédroits et parfois de venues d'eau.

La microfissuration pédogénétique de certaines marnes a posé quelques problèmes de tenue, en calotte surtout. Les rares et peu importantes venues d'eau rencontrées n'ont causé que des problèmes mineurs.

Les problèmes de gonflement dans les marnes constituent de loin les difficultés les plus importantes rencontrées pendant et surtout après la creuse du tunnel, avec les problèmes de déformation du revêtement et du plan de roulement. 
Tableau 2. Descriptions des carottes, classes de Molasse y compris celles de l'étude géologique.

Table 2. Boring description, Molasse classes including those from the geological study.

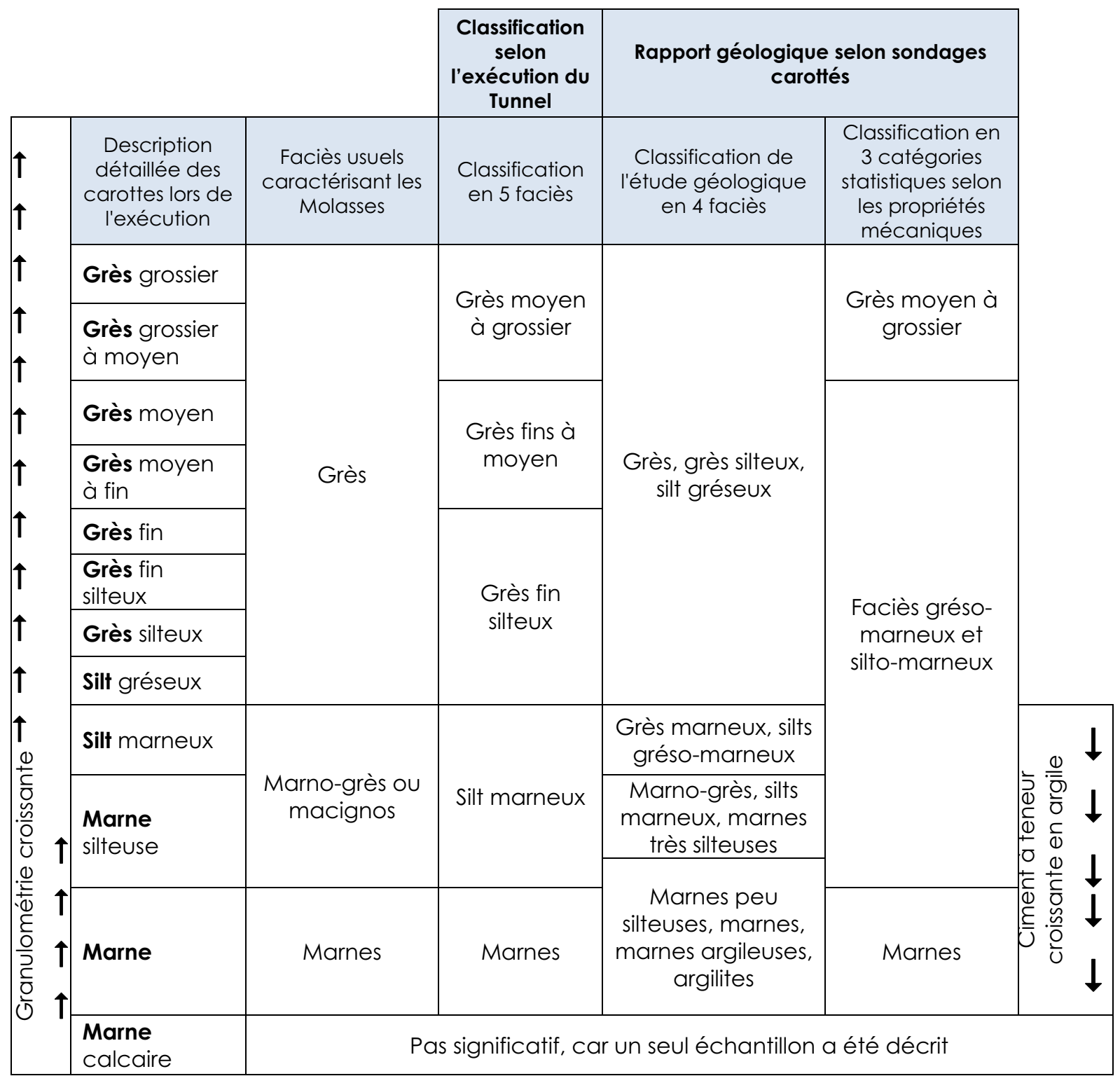

\section{Corrélations entre les types lithologiques de la molasse et les propriétés mécaniques}

L'ensemble des résultats obtenus à partir des essais effectués sur les 5 faciès de Molasse sont résumés dans le tableau 3. Ce tableau montre pour chaque type de faciès la valeur moyenne ainsi que l'écart-type, le nombre d'essais et les valeurs extrêmes des caractéristiques mécaniques déterminées en laboratoire, à savoir la résistance uniaxiale $\sigma_{c}$, la résistance à la traction $\sigma_{t}$ et le module d'élasticité $E$. Les valeurs de $\sigma_{t}$ ont été obtenues à partir d'essais brésiliens. Le module $E$ a été déterminé lors des essais de compression simple au palier de contrainte correspondant à $50 \%$ de la résistance à la rupture.

L'échelle des résistances que présentent les différents faciès lithologiques de la Molasse Grise de Lausanne est très large, puisque des valeurs $\sigma_{c}$ extrêmes allant de moins de $0,2 \mathrm{MN} / \mathrm{m}^{2}$ (argilites, marnes argileuses) ponctuellement à plus de $75 \mathrm{MN} / \mathrm{m}^{2}$ dans certains silts et grès très fins à ciment calcaire abondant ont été mesurées. Les faciès gréseux à grains grossiers sont situés dans la fourchette inférieure de ces résistances, avec des valeurs parfois inférieures à $5 \mathrm{MN} / \mathrm{m}^{2}$.

Le nombre d'essais de compression uniaxiale effectué dans les marnes est resté peu élevé, en raison du caractère 
Tableau 3. Propriétés mécaniques des 5 faciès de Molasse.

Table 3. Mechanical properties for 5 types of Molasse.

\begin{tabular}{|c|c|c|c|c|c|}
\hline $\begin{array}{l}\text { Molasse aquitanienne de Lausanne: } \\
\text { Type de faciès lithologique }\end{array}$ & & $\begin{array}{l}\text { Résistance } \\
\text { uniaxiale } \\
\sigma_{c}\left[\mathrm{MN} / \mathrm{m}^{2}\right]\end{array}$ & $\begin{array}{l}\text { Résistance à } \\
\text { la traction } \\
\sigma_{t}\left[\mathrm{MN} / \mathrm{m}^{2}\right]\end{array}$ & $\begin{array}{l}\text { Module } \\
\text { d'Elasticité } \\
E\left[\mathrm{MN} / \mathrm{m}^{2}\right]\end{array}$ & $\begin{array}{l}\text { Masse } \\
\text { volumique } \\
{\left[\mathrm{t} / \mathrm{m}^{3}\right]}\end{array}$ \\
\hline \multirow{3}{*}{ Grès grossier à moyen } & Écart-type & 6,26 & 0,33 & 1890 & \multirow{3}{*}{2,41} \\
\hline & Nombre & 30 & 30 & 25 & \\
\hline & Min-max & $4,7-33,2$ & $0,19-1,28$ & $900-9500$ & \\
\hline \multirow{3}{*}{ Grès moyen à fin } & Écart-type & 7,56 & 0,5 & 2069 & \multirow{3}{*}{2,46} \\
\hline & Nombre & 89 & 89 & 79 & \\
\hline & Min-max & $5,6-40,8$ & $0,26-3,10$ & $800-10700$ & \\
\hline Grès fin silteux & Moyenne & 32,9 & 2,42 & 7281 & 2,60 \\
\hline \multirow{4}{*}{ Silt marneux } & Moyenne & 14,00 & 1,22 & 2754 & \multirow{4}{*}{2,56} \\
\hline & Écart-type & 8,03 & 0,63 & 3775 & \\
\hline & Nombre & 42 & 42 & 36 & \\
\hline & Min-max & $3,2-40,2$ & $0,13-2,66$ & $500-8600$ & \\
\hline \multirow{4}{*}{ Marne } & Moyenne & 3,30 & 0,46 & 615 & \multirow{4}{*}{2,43} \\
\hline & Écart-type & 2,61 & 0,28 & 471 & \\
\hline & Nombre & 21 & 21 & 16 & \\
\hline & $\operatorname{Min}-\max$ & $0,2-8,5$ & $0,03-1,04$ & $50-2050$ & \\
\hline
\end{tabular}

fréquemment lité et microfissuré de ces roches qui empêchait un carottage correct. De même pour les marno-grès, déjà faiblement représentés, et qui se prêtaient mal au carottage.

Les valeurs moyennes des masses volumiques mesurées figurent pour les différentes catégories de faciès dans le tableau 3. Les paramètres statistiques ne sont pas indiqués car les valeurs obtenues montrent une très faible dispersion avec un coefficient de variation inférieur à $2 \%$.

Les masses volumiques sont toutes inférieures à celles des minéraux constitutifs de la roche, tant ceux du ciment que ceux de la partie granulaire. La différence provient de la présence de vides entre grains et ciment. Ces vides sont plus abondants dans les faciès grossiers que dans les faciès granulaires fins (siltites).

Les valeurs basses pour les marnes sont dues surtout à la faible densité des minéraux des argiles les plus hydratés.

La porosité des grès grossiers due à la répartition incomplète du ciment calcique est l'une des causes de la plus faible résistance à la compression de ces grès par rapport aux faciès plus fins. L'autre raison tient à la liaison médiocre entre les grains de minéraux silicatés et le ciment calcique.

Les résistances les plus élevées ont été obtenues dans le cas des grès fins silteux qui contiennent vraisemblablement le pourcentage le plus élevé en ciment calcique.

Les valeurs des paramètres obtenus en cours d'exécution sont totalement compatibles avec celles déterminées pour les différentes catégories de Molasse sur un nombre relativement restreint d'échantillons prélevés en forages lors des études géologiques préliminaires.

La variabilité des résultats des essais de compression simple est très grande également pour chaque type de faciès lithologique, pour des raisons qui ne sont probablement pas toujours en relation avec les propriétés intrinsèques de la roche testée (microfissures non repérées, altération plus ou moins développée, description lithologique peut-être parfois légèrement inexacte de l'échantillon, carottage soumis à l'essai effectué tantôt perpendiculairement tantôt parallèlement au litage, etc.).

Le coefficient de variation des résultats est compris en général entre 40 et $60 \%$ environ; ce coefficient est de l'ordre de $76 \%$ dans le cas des marnes. De ce fait, et en supprimant les valeurs extrêmes qui n'ont vraisemblablement pas à être prises en compte, les fourchettes de valeurs $\sigma_{c}$ et $\sigma_{t}$ des différents faciès sont assez significatives. Les valeurs moyennes des différents faciès sont représentées sur la figure 2 .

Il ressort de ces résultats que tous les faciès lithologiques à grains fins, y compris les silts à faible composante marneuse, présentent des valeurs moyennes de résistance à la compression très semblables, mais globalement près de deux fois plus élevées que les grès grossiers à moyens. Par contre, les valeurs $\sigma_{c}$ très élevées $\left(50-84 \mathrm{MN} / \mathrm{m}^{2}\right)$ ont été constatées uniquement dans les grès fins silteux et les silts gréseux, mais elles ne concernent que 3 échantillons. 


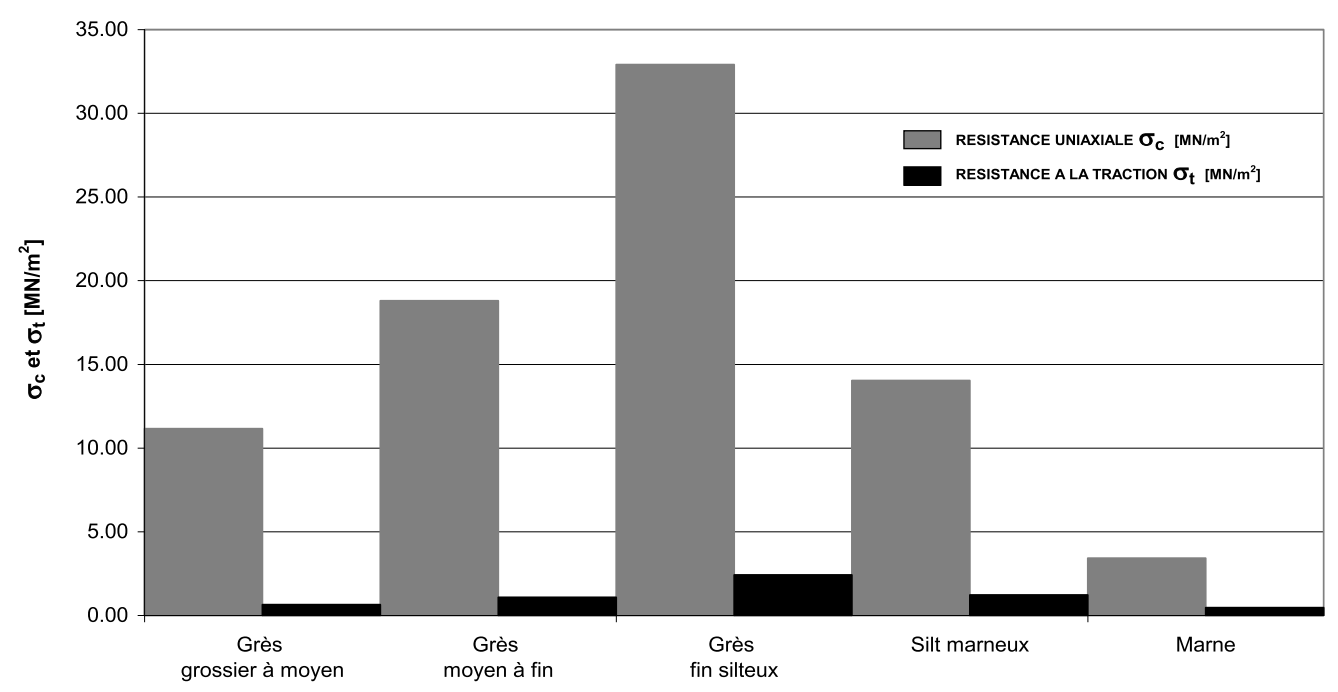

Fig. 2. Valeurs moyennes des résistances uniaxiales et à la traction pour les 5 faciès de Molasse.

Fig. 2. Average uniaxial strength and tensile strength values for 5 types of Molasse.

\section{CORRELATION RESISTANCE UNIAXIALE $\sigma_{c}$ - TRACTION $\sigma_{t}$}

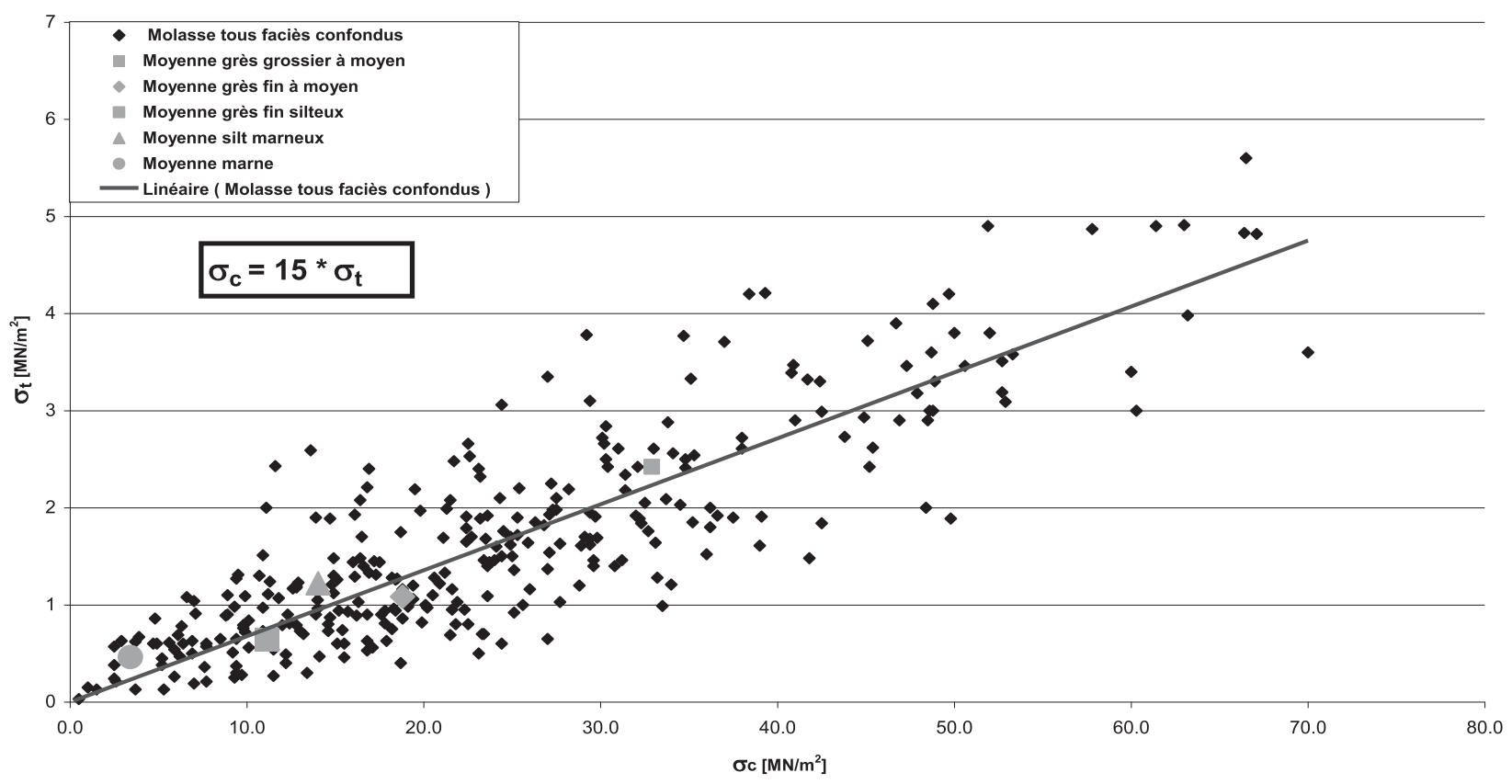

Fig. 3. Relation entre $\sigma_{c}$ et $\sigma_{t}$ pour tous les faciès de Molasse confondus.

Fig. 3. Relationship between $\sigma_{c}$ and $\sigma_{t}$ i for all types of Molasse.

\section{Corrélation entre les caractéristiques mécaniques des différents types de molasse}

\subsection{Relation $\sigma_{c}$ et $\sigma_{t}$}

La figure 3 illustre la corrélation entre la résistance uniaxiale $\sigma_{c}$ et la résistance à la traction $\sigma_{t}$ pour tous les faciès confondus de la Molasse aquitanienne.
La droite de régression définie sur la base des résultats obtenus indique une relation linéaire entre $\sigma_{c}$ et $\sigma_{t}$ :

$$
\sigma_{c}=15 \cdot \sigma_{t}
$$

La qualité de la corrélation est bonne (coefficient de corrélation $\mathrm{R}=0,83$ ). On peut aussi constater sur la figure 3 que les valeurs moyennes de chaque type de 
CORRELATION MODULE D'ELASTICITE E - RESISTANCE UNIAXIALE $\sigma_{\mathrm{c}}$

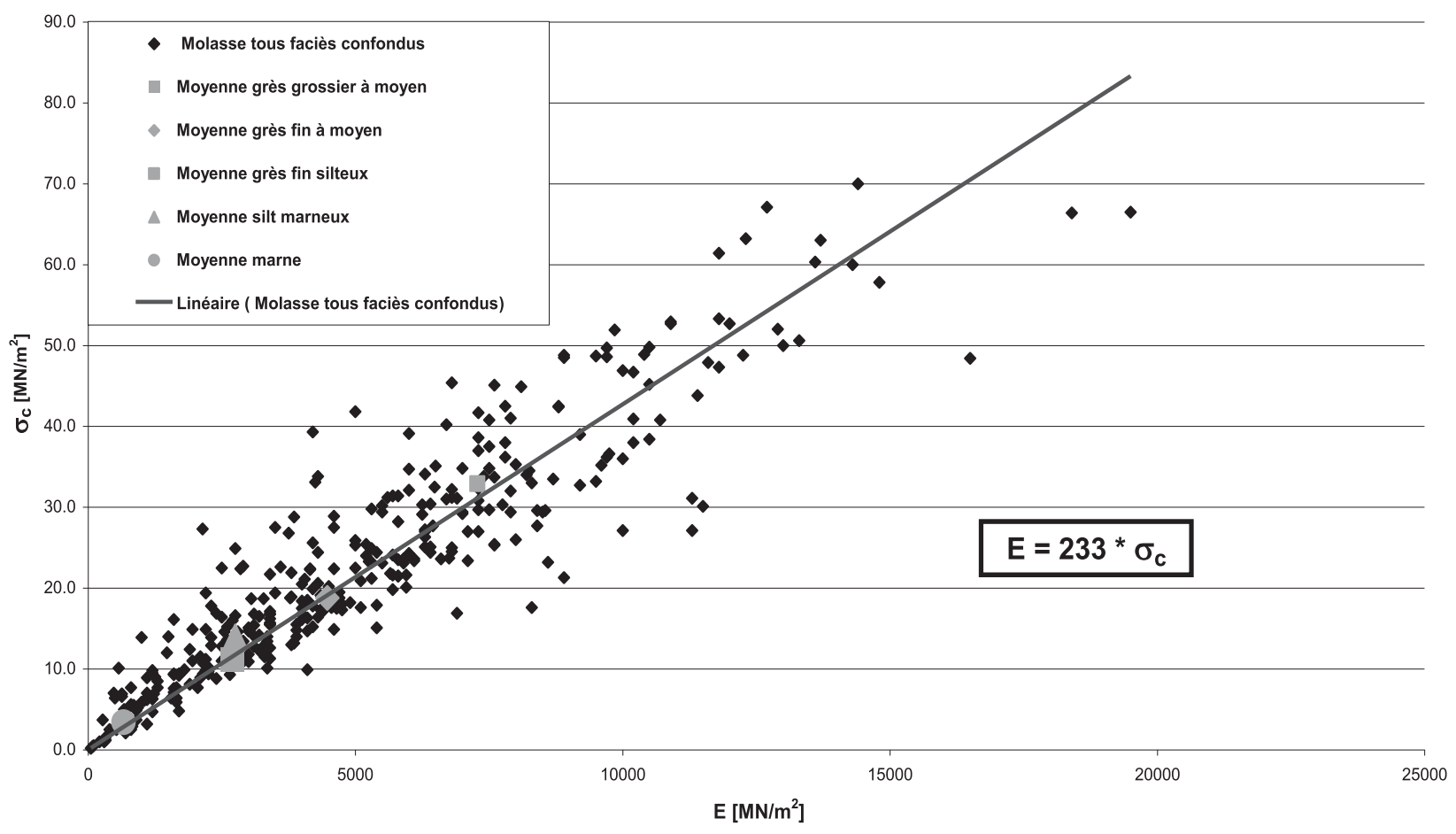

Fig. 4. Relation linéaire entre $\sigma_{c}$ et module $E$ pour tous les faciès de Molasse.

Fig. 4. Linear relationship between $\sigma_{c}$ and $E$ modulus for all types of Molasse.

Molasse montrent une parfaite corrélation du fait que celles-ci sont situées sur la même droite de régression. Toutefois, on constate une très grande dispersion des résultats quand on considère individuellement les catégories définies. La représentation graphique par catégorie qui ne présente pas d'intérêt particulier, n'a pas été jugée utile dans le cadre de cet article.

On peut toutefois préciser que l'équation (1) n'est pas confirmée statistiquement dans le cas des grès grossiers et des marnes à cause de leur faible teneur en ciment calcaire et de leur structure particulière (microfissuration aléatoire pour les marnes). En effet, les résultats obtenus montrent une dispersion importante. Il convient également de préciser qu'un nombre réduit d'échantillons a pu être prélevé par carottage dans ces deux catégories de Molasse. L'équation (1) est plutôt confirmée dans le cas des grès moyens à fins, des grès fins silteux et des silts marneux.

Il est à noter que le rapport $m=\sigma_{c} / \sigma_{t}$ appelé l'indice de fragilité est une propriété physique qui varie selon le type de roche. Ce paramètre est utilisé pour définir les critères de rupture de la roche intacte comme décrit dans la section 9.

\subsection{Relation $\sigma_{c}-E$}

La figure 4 illustre la corrélation entre la résistance uniaxiale $\sigma_{c}$ et le module d'élasticité $E$ pour tous les faciès de Molasse confondus.

La corrélation entre ces deux propriétés mécaniques est très bonne (coefficient de corrélation $\mathrm{R}=0,92$ ) ce qui permet de définir la relation suivante:

$$
E=233 \cdot \sigma_{c}
$$

On peut constater également que les valeurs moyennes des différents faciès de Molasse sont situées sur la droite de régression de la figure 4.

L'équation (2) est également confirmée avec une très bonne corrélation dans le cas des grès en général et des grès fins silteux comme l'indique les figures 5 et 6 .

D'une manière générale le rapport du module d'élasticité sur la résistance uniaxiale est de l'ordre de 230 à 250 pour tous les types de grès qui ne comportent pas de composants marneux.

En revanche, dans le cas des silts marneux et des marnes pour lesquels le teneur en ciment marneux augmente, on constate qu'il n'existe pas de corrélation nette entre $\sigma_{c}$ et $E$ du fait de la forte dispersion des résultats.

\section{Essais triaxiaux sur les marnes}

Lors de l'exécution du tunnel, quatre échantillons de marne ont été prélevés par carottage sous le radier du tunnel dans les zones où des gonflements ont été observés.

Ces échantillons ont été soumis à des essais triaxiaux selon le mode opératoire préconisé par la Société Internationale de Mécanique des Roches (ISRM, 1983). Il s'agit d'un essai type III durant lequel l'échantillon est sollicité continuellement à 


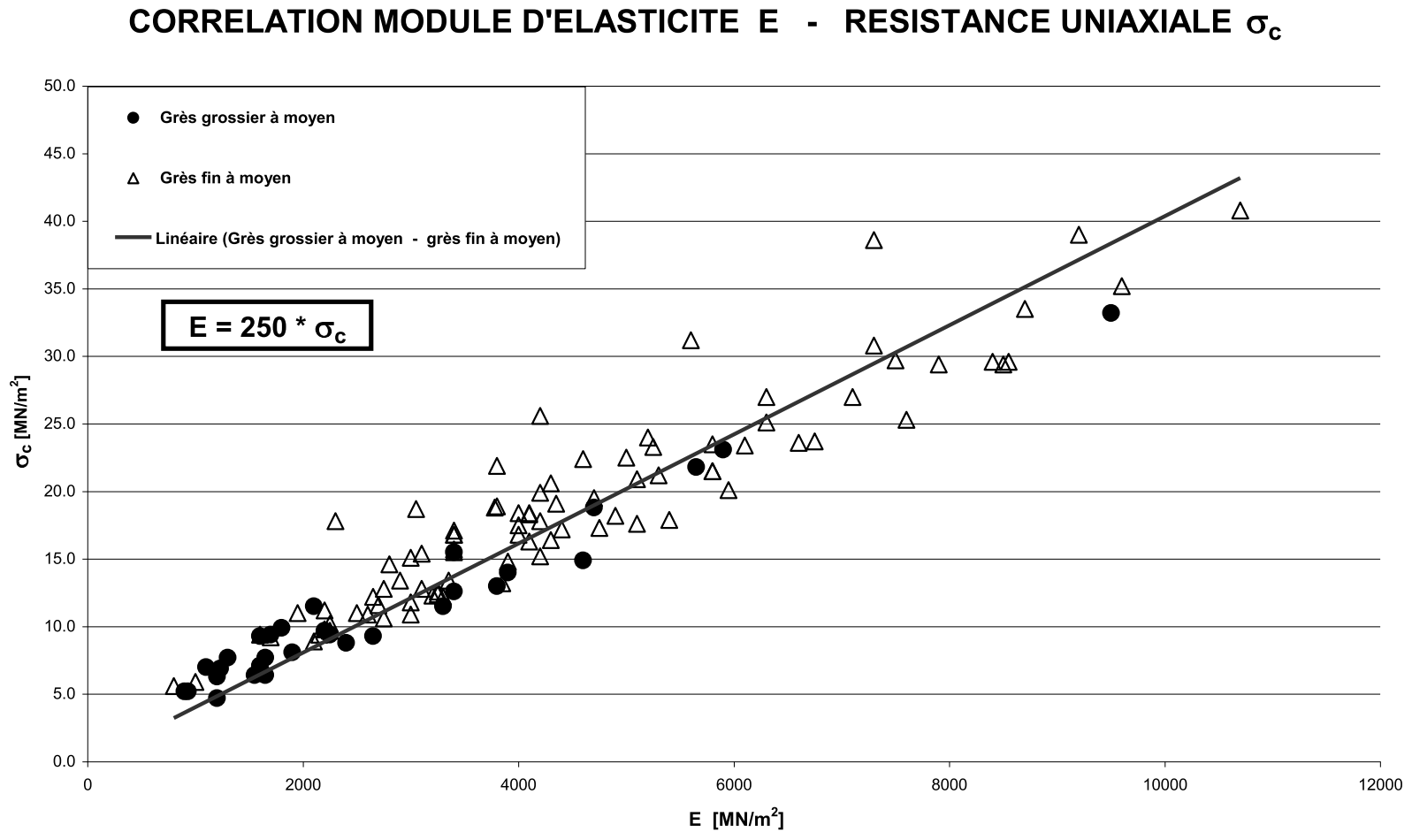

Fig. 5. Corrélation $\sigma_{c}-E$ pour les grès grossiers à fins.

Fig. 5. Correlation $\sigma_{c}-E$ for coarse to fine sandstone.

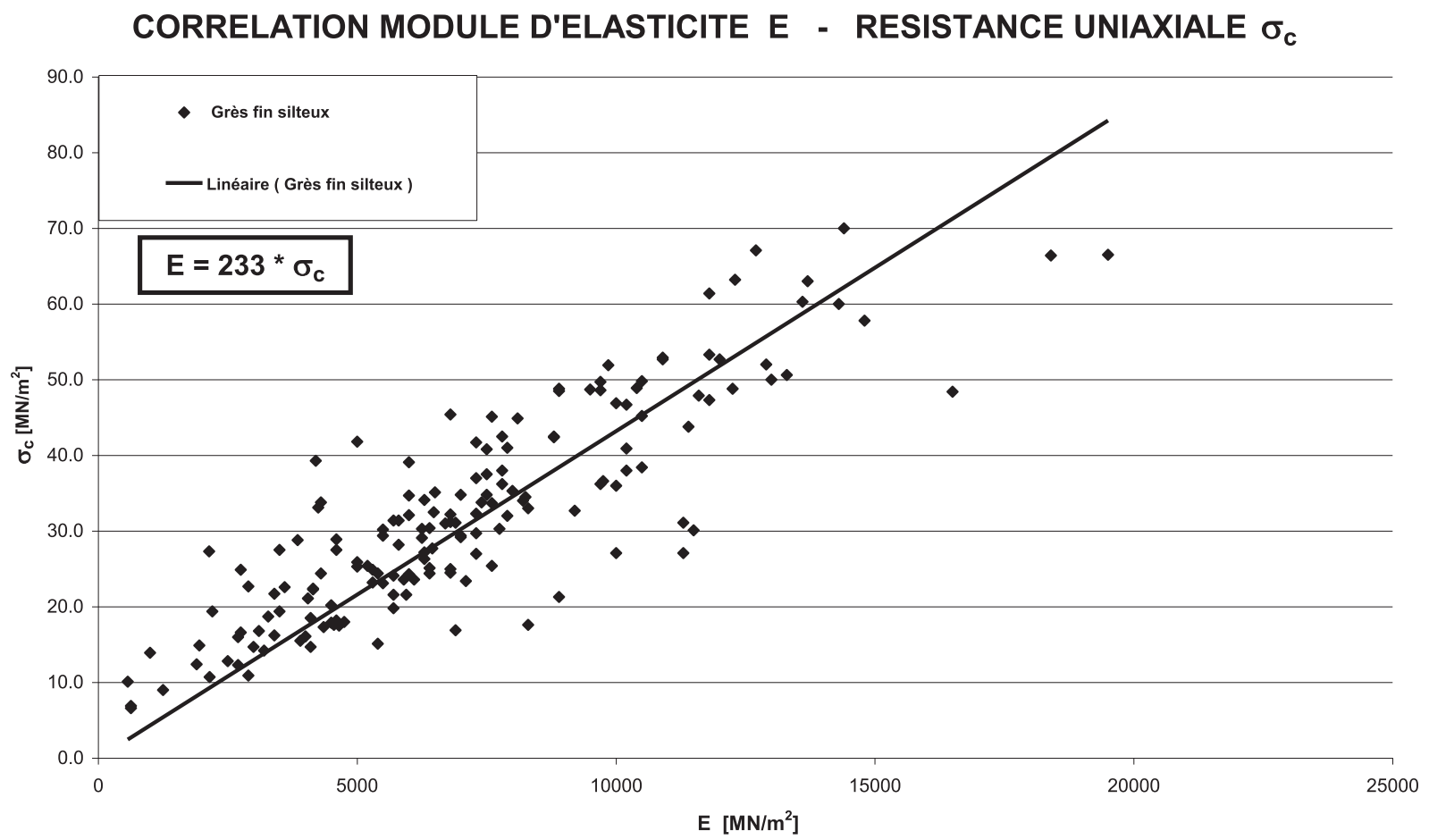

Fig. 6. Corrélation $\sigma_{c}-E$ pour les grès fins silteux.

Fig. 6. Correlation $\sigma_{c}-E$ for silty fine sandstone. 

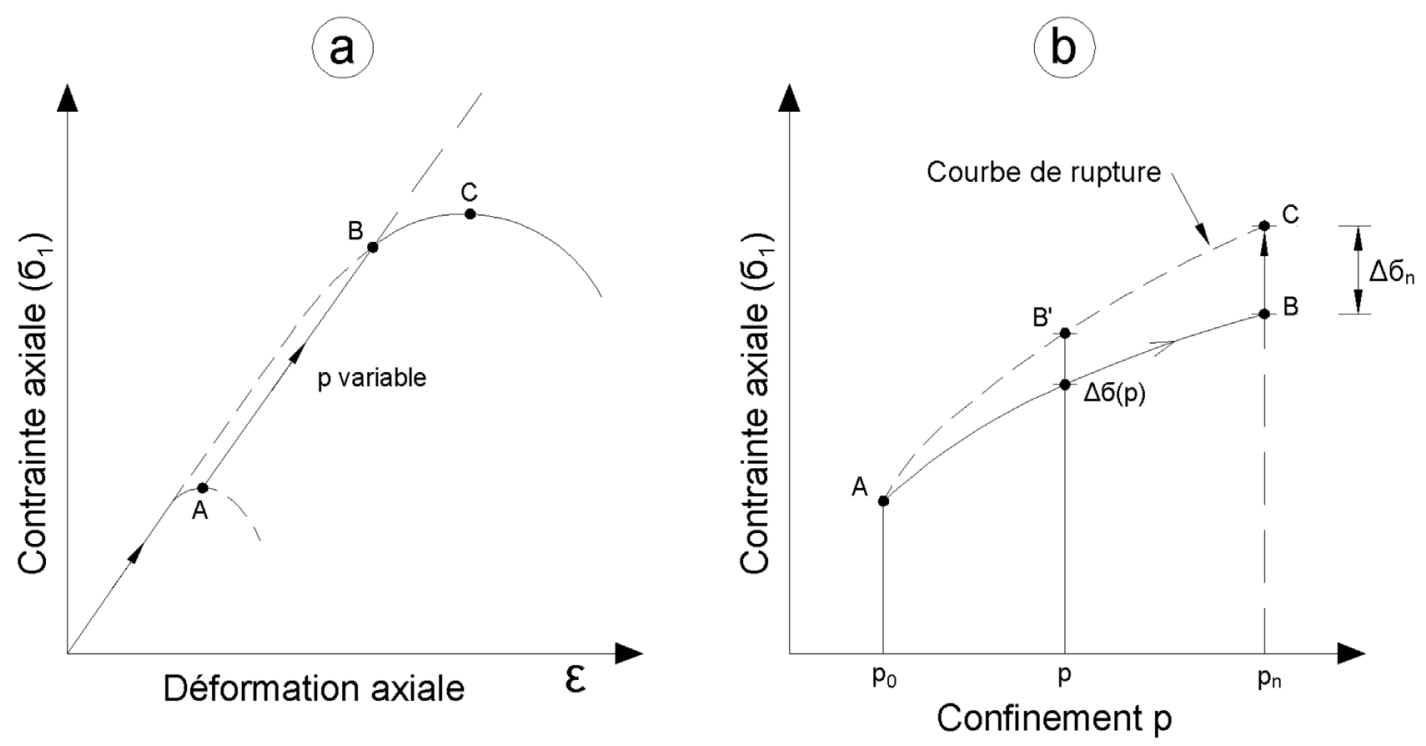

Fig. 7. (a) Courbe de contraintes et de déformations axiales. (b) Chemin des contraintes principales $\sigma_{1}$ et $\sigma_{3}$.

Fig. 7. (a) Stress and axial deformation curve. (b) Principal stress paths $\sigma_{1}$ and $\sigma_{3}$.

l'état de rupture pour pouvoir obtenir la courbe intrinsèque de résistance de la roche à l'aide d'un seul essai triaxial.

Les figures $7 \mathrm{a}$ et $\mathrm{b}$ illustrent de manière qualitative les résultats obtenus lors d'un essai triaxial.

L'essai débute en appliquant une contrainte de confinement $p_{o}=\sigma_{3}$ constante à l'échantillon. Le point $\mathrm{A}$ est obtenu en augmentant la contrainte axiale $\sigma_{1}$ jusqu'à la résistance de pic. Ensuite, on augmente simultanément les contraintes axiales et de confinement jusqu'à un niveau correspondant au point $\mathrm{B}$ choisi. À partir de ce point, la contrainte de confinement est maintenue constante et la contrainte axiale est augmentée pour atteindre la rupture correspondant au point C. Lors de l'essai, les déformations axiales sont également mesurées. Les valeurs de contrainte de confinement $\sigma_{3}$ appliquées lors des essais varient entre 0,5 et $4 \mathrm{MN} / \mathrm{m}^{2}$.

Le chemin de contrainte entre A et B est corrigé en tenant compte de la réserve de résistance $\Delta \sigma_{n}$ entre les points $\mathrm{B}$ et $\mathrm{C}$ comme indiqué sur la figure $6 \mathrm{~b}$ pour obtenir la courbe intrinsèque de rupture. Nous avons donc défini un point intermédiaire B' en appliquant la correction proposée selon le mode opératoire de l'ISRM. Nous avons donc retenu trois couples de valeurs de $\left(\sigma_{3}, \sigma_{1}\right)$ pour chaque échantillon (points $\mathrm{A}, \mathrm{B}$ ' et $\mathrm{C}$ ) afin de définir les paramètres des critères de rupture pour les marnes.

\section{Lois de rupture pour la molasse marneuse}

\subsection{Critère de rupture Hoek-Brown}

Ce critère empirique est couramment utilisé en mécanique des roches du fait qu'il est bien adapté pour décrire le comportement de la roche intacte et du massif rocheux à grande échelle.

Le critère de Hoek-Brown (1980 et 2002) a subi de nombreuses modifications dès son apparition en 1980. Dans sa forme originale, ce critère peut être exprimé en fonction des contraintes principales $\sigma_{1}$ majeure et $\sigma_{3}$ mineure comme suit :

$$
\sigma_{1}=\sigma_{3}+\sigma_{c}\left[\left(m . \sigma_{3} / \sigma_{c}\right)+s\right]^{1 / 2}
$$

où $\sigma_{c}$ correspond à la résistance uniaxiale et les paramètres $m$ et $s$ dépendent du type de roche. Dans le cas de la roche intacte, on a $s=1$ et le paramètre $m$ varie entre 7 et 30 en fonction de la nature de la roche considérée. Ce dernier paramètre $m$ doit être déterminé à partir des résultats d'essais triaxiaux sur les échantillons de roche intacte.

Selon AFTES (2003) et les travaux récents de Sari (2010), le paramètre $m$ est très voisin de l'indice de fragilité exprimé comme suit :

$$
m=\frac{\sigma_{c}}{\sigma_{t}}
$$

Cette hypothèse permet d'attribuer au paramètre $m$ un sens physique et de définir avec une très bonne approximation le critère de rupture de Hoek \& Brown. Dans le cas de la Molasse aquitanienne, le paramètre $m$ est proche de 15 . Il est à noter que ce paramètre peut varier fortement dans le cas des grès grossiers mal cimentés et des marnes. En effet, pour ces types de matériaux, les valeurs de la résistance à la traction déterminées à l'aide d'essais brésiliens montrent une très grande dispersion. La valeur de $m$ est nettement inférieure à 15 notamment pour les marnes, pour lesquelles elle est comprise entre 5 et 10 environ.

Pour chaque échantillon, les trois couples de valeurs de $\sigma_{1}$ et $\sigma_{3}$ correspondant aux points A, B' et $\mathrm{C}$ du chemin des contraintes ont été retenus pour définir le critère de rupture. Les résultats sont reportés sur la figure 8 .

Pour définir de manière indirecte les paramètres $m$ et $\sigma_{c}$, Hoek \& Brown (1980) ont proposé une méthode statistique en utilisant les résultats d'essais triaxiaux qui permet d'obtenir la courbe intrinsèque de rupture selon l'équation (3). 


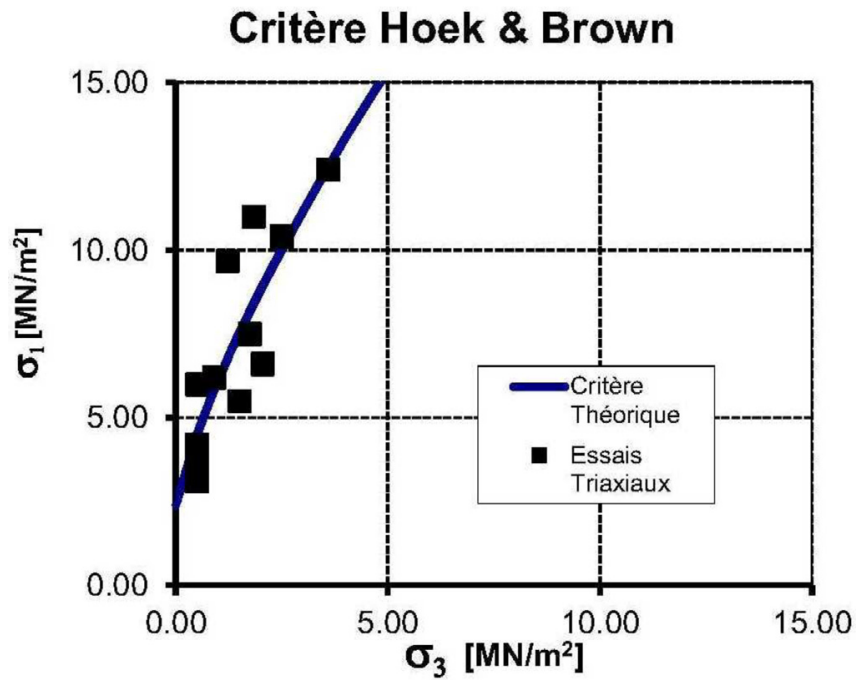

Fig. 8. Résultats des essais triaxiaux et le critère de Hoek-Brown. Fig. 8. Triaxial tests results and Hoek-Brown criteria.

La courbe pleine sur la figure 8 représente la loi de rupture selon Hoek-Brown définie, en admettant

$\sigma_{c}=2,4 \mathrm{MN} / \mathrm{m}^{2}$ et $m=8,5$ qui sont des paramètres dont les valeurs ont été calculées à partir des résultats des essais triaxiaux. On peut constater que les résultats des essais confirment avec une bonne concordance la courbe théorique de rupture.

Il est à relever que les valeurs de ces paramètres déduits des essais triaxiaux sont sensiblement proches de celles qui représentent la moyenne de la résistance uniaxiale des marnes, soit $\sigma_{c}=3,3 \mathrm{MN} / \mathrm{m}^{2}$ et $m=7,2$ (indice de fragilité), ce dernier correspond au rapport des valeurs moyennes de $\sigma_{c}$ et $\sigma_{t}$ déterminées sur les marnes (voir Tab. 3).

L'équation (3) peut être utilisée dans le cadre des projets d'ouvrages souterrains dans la Molasse aquitanienne essentiellement gréseuse et silteuse pour estimer le comportement à la rupture de la Molasse en admettant $m=15$ (ou entre 5 et 10 pour les marnes) et $s=1$ sur la base d'un seul paramètre $\sigma_{c}$ qui caractérise la résistance uniaxiale d'un faciès donné. Cette hypothèse a été validée dans le cas de la Molasse marneuse sur la base des résultats des essais triaxiaux effectués sur les échantillons de marnes.

\subsection{Critère de Mohr-Coulomb et de Drucker-Prager, application du principe d'état critique}

Ce critère peut être considéré comme une linéarisation de la courbe intrinsèque de rupture. En pratique, la plupart des modèles de calculs numériques utilisent encore ce critère pour modéliser la résistance de la roche intacte.

Par analogie avec la mécanique des sols, la résistance à la rupture des roches est exprimée souvent selon le critère de Mohr-Coulomb dans les modèles de calculs numériques.

Ce critère est défini sous sa forme la plus connue par une relation linéaire en fonction des paramètres $\phi$ (angle de frottement interne) et $c$ (cohésion de la roche).

$$
\tau=c+\sigma \cdot \tan \varphi
$$

où $\tau$ et $\sigma$ représentent respectivement la résistance au cisaillement et la contrainte normale sur le plan de rupture. Le comportement à la rupture des roches n'est pas linéaire, il est exprimé en général par une loi parabolique.

Le critère de Mohr-Coulomb est applicable à certaines roches notamment très fracturées ou dans une plage limitée de contraintes en linéarisant une relation parabolique.

Le critère de Mohr-Coulomb peut être également décrit dans le plan des contraintes principales $\sigma_{1}$ et $\sigma_{3}$ sous la forme linéaire suivante :

$$
\sigma_{1}=\sigma_{c}+\sigma_{3} \cdot K_{p}
$$

où

$$
K_{p}=\tan ^{2}\left(45^{\circ}+\frac{\varphi}{2}\right)
$$

Il est à noter que le terme $K_{p}$ représente le coefficient de poussée passive (butée) selon la théorie de Rankine en mécanique des sols.

Par analogie avec les modèles d'état critique en mécanique des sols, les résultats des essais triaxiaux peuvent être représentés dans le plan de contrainte moyenne $p$ et de déviateur $q$ comme indiqué sur la figure 9. Dans ce type de représentation, on obtient en général une droite dont la pente est caractérisée par le paramètre $M_{p}$ comme dans le cas du modèle de Cam-Clay. Le paramètre $C_{m}$ est obtenu par l'intersection de cette droite avec l'axe de déviateur des contraintes.

Le paramètre $c$ et $\phi$ du critère de Mohr-Coulomb sont ensuite définis comme suit:

$$
\begin{gathered}
\varphi=\operatorname{Arcsin} \frac{3 M_{p}}{\left(6+M_{p}\right)} \\
c=\frac{3-\sin \varphi}{(6 \cos \varphi)} \cdot C_{m}
\end{gathered}
$$

Les résultats des essais triaxiaux correspondant aux points $\mathrm{A}, \mathrm{C}$ et B' (Fig. 7) ont été retenus pour définir le critère de Mohr-Coulomb. La figure 10 présente les résultats dans le plan $p$ et $q$ selon le concept d'état critique et les paramètres de la droite de régression (droite intrinsèque de rupture) obtenue avec un coefficient de corrélation très satisfaisant de $\mathrm{R}=0,89$.

On constate donc que dans le cas des marnes, le critère de Mohr-Coulomb traduit assez bien le comportement à la rupture de ce type de Molasse par une relation linéaire.

Le critère de Drucker-Prager est très largement utilisé lors des simulations numériques par éléments finis. Le succès de ce critère est dû essentiellement à sa facilité d'implémentation dans les programmes d'éléments finis. En fait, le critère de Drucker-Prager n'est pas mieux adapté par rapport aux critères de Mohr-Coulomb et de Hoek-Brown pour représenter le comportement des roches. Les paramètres $k$ et $\alpha$ de ce critère sont également proposés dans le présent article. Le critère de Drucker-Prager est exprimé par la relation suivante :

$$
q=3 \sqrt{3} \cdot \sigma \cdot p+\sqrt{3} \cdot k
$$




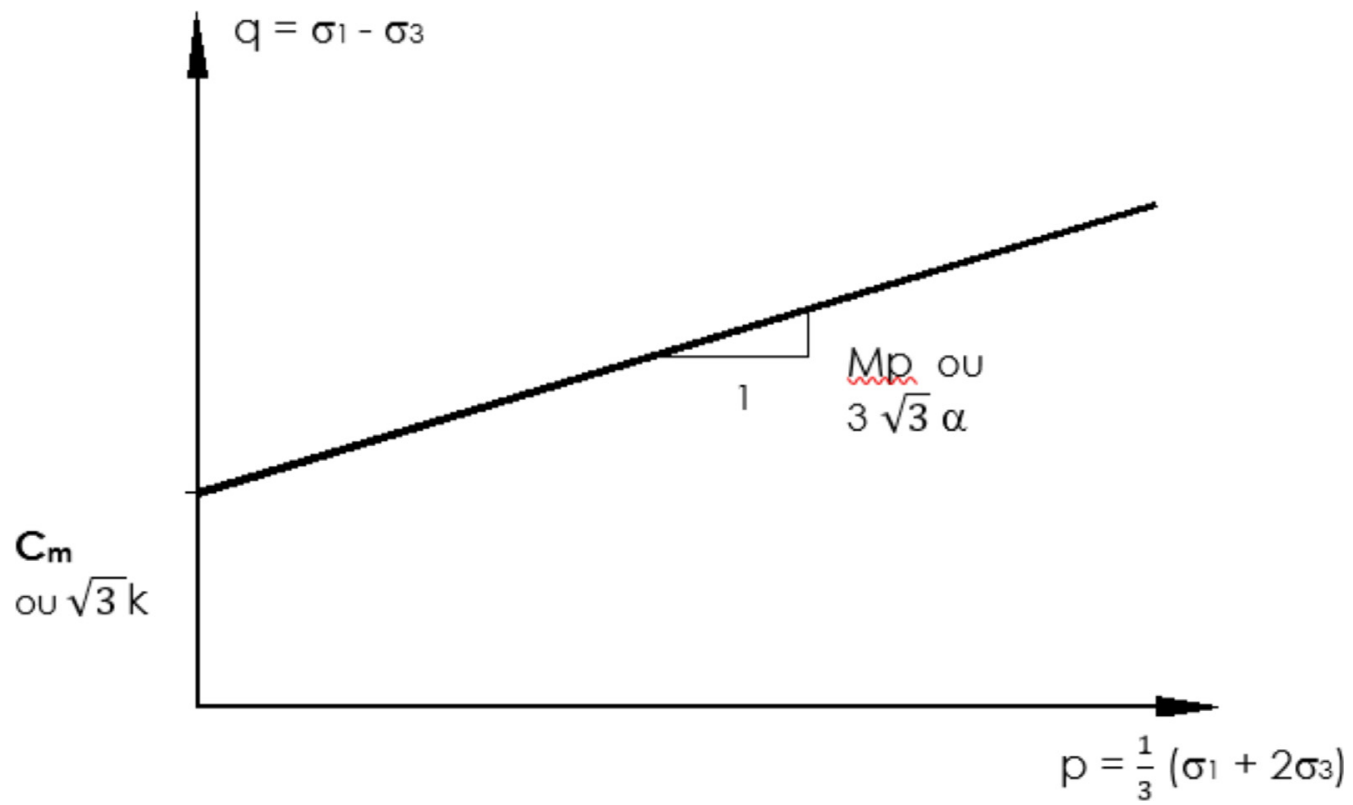

Fig. 9. Détermination des paramètres du critère Mohr-Coulomb et de Drucker-Prager dans un essai triaxial.

Fig. 9. Determination of parameters for the Mohr-Coulomb and Drucker-Prager models from triaxial test results.

Tableau 4. Paramètres de Mohr-Coulomb pour les marnes.

Table 4. Mohr-Coulomb parameters for claystone.

\begin{tabular}{llllll}
\hline$M_{p}[-]$ & $C_{m}\left[\mathrm{MN} / \mathrm{m}^{2}\right]$ & Angle de frottement $\phi\left[^{\circ}\right]$ & Cohésion $c\left[\mathrm{MN} / \mathrm{m}^{2}\right]$ & $\alpha[-]$ & $k\left[\mathrm{MN} / \mathrm{m}^{2}\right]$ \\
\hline 1,29 & 1,39 & 32 & 0,67 & 0,25 & 0,80 \\
\hline
\end{tabular}

\section{Critère de Mohr-Coulomb}

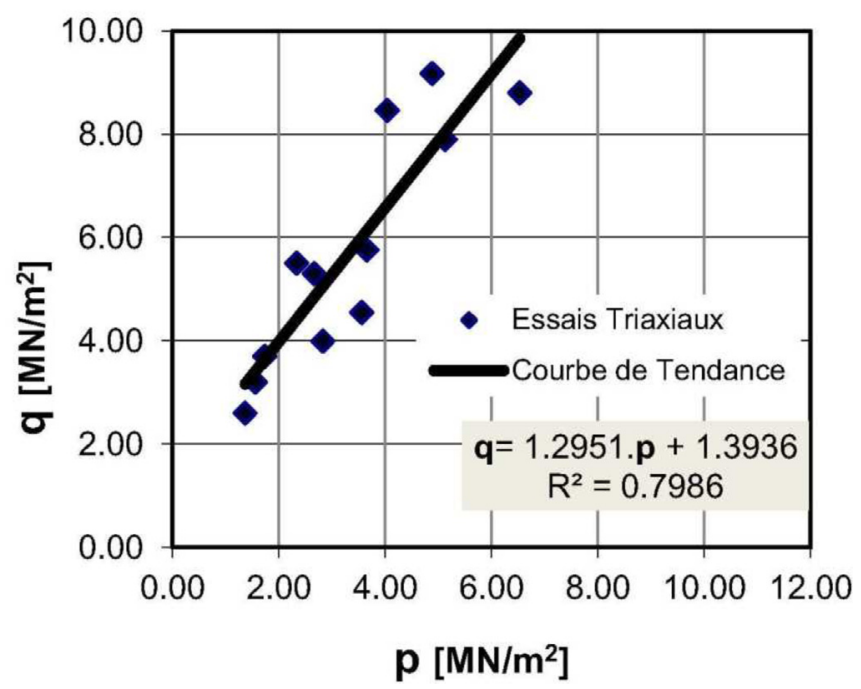

Fig. 10. Le critère de Mohr-Coulomb et les résultats des essais triaxiaux.

Fig. 10. Mohr-Coulomb failure criteria and triaxial test results.
Les valeurs des paramètres déduites des essais triaxiaux sont résumées dans le tableau 4.

L'angle de frottement obtenue $\varphi=32^{\circ}$ semble a priori assez élevé pour des marnes qui sont essentiellement des roches argileuses. Ce résultat est dû certainement à l'effet d'imbrication ou de dilatance que l'on observe quand la résistance de pic est atteinte lors de l'essai. La résistance uniaxiale peut être déduite à partir de la cohésion $c=0,67 \mathrm{MN} / \mathrm{m}^{2}$ par la relation conformément au critère de Mohr-Coulomb.

$$
\sigma_{c}=\frac{2 \cdot c \cdot \cos \varphi}{1-\sin \varphi}
$$

La résistance uniaxiale obtenue est de $\sigma_{c}=2,4 \mathrm{MN} / \mathrm{m}^{2}$, ce qui est pratiquement identique à la valeur déduite ci-avant selon le critère de Hoek \& Brown.

Comme dans le cas du critère de Hoek \& Brown, le critère de rupture de la roche intacte selon Mohr-Coulomb peut être aussi exprimé sur la base de sa résistance uniaxiale $\sigma_{c}$. (voir l'Éq (6)). Le cas échéant l'angle de frottement interne $\varphi$ qui dépend de la composition granulométrique de la roche, pourra être défini sur la base des essais de cisaillement direct, moins onéreux que les essais triaxiaux. 


\section{Conclusions}

Le tunnel TRIDEL est le premier tunnel réalisé à relativement grande profondeur dans la Molasse aquitanienne, en l'occurrence à plus de $70 \mathrm{~m}$ depuis la surface sur certains tronçons du tracé. Les roches constituant cette Molasse donnent naissance à la formation de différents faciès lithologiques définis selon leur composition granulométriques. Les relevés géologiques lors de l'exécution de ce tunnel ainsi que l'examen des 350 échantillons prélevés au front d'excavation par carottage ont permis de décrire lithologiquement les différents faciès de la Molasse. Sur la base des critères lithologiques (granulométrie des grains et nature du ciment argileux et calcaire), et des caractéristiques géomécaniques, les faciès molassiques ont été regroupés en 5 classes distinctes.

Les propriétés géomécaniques (résistance uniaxiale, à la traction, ainsi que l'indice de fragilité et le module d'élasticité) ont été définies pour chaque type de faciès à partir des essais de compression simple et des essais brésiliens. Les corrélations entre les différentes propriétés ont été analysées afin de définir les paramètres de la loi empirique du comportement à la rupture des roches selon les critères de Hoek-Brown et de Mohr-Coulomb dans le cas de la Molasse aquitanienne. Ces deux critères, couramment utilisés dans les calculs numériques pour modéliser le comportement à la rupture de la roche, peuvent être exprimés en fonction de la résistance à la compression uniaxiale pour un type de faciès donné de la Molasse.

Le critère de Hoek-Brown est entièrement défini avec une bonne approximation en admettant que le paramètre $m$ spécifique à ce critère correspond à l'indice de fragilité de la roche qui représente le rapport de la résistance uniaxiale et de la traction. Les valeurs de cet indice pour toutes les catégories confondues de la Molasse aquitanienne sont obtenues sur la base des résultats d'essais de compression simple et brésiliens.
Le terme de cohésion dans le critère de Mohr-Coulomb est remplacé par la résistance à la compression uniaxiale d'un faciès donné. L'angle de frottement interne est estimé en fonction des caractéristiques minéralogiques. Ce paramètre peut être aussi déterminé sur la base d'essais de cisaillement direct moins onéreux que les essais triaxiaux, effectués par exemple sur des surfaces planes sciées dans la roche.

Les valeurs moyennes des paramètres géomécaniques peuvent être utilisées pour définir les critères de rupture. Malgré une grande dispersion des valeurs de $\sigma_{c}$ et $\sigma_{t}$ obtenues dans le cas des marnes, les essais triaxiaux confirment la validité des critères de rupture ainsi définis.

Remerciements. Les auteurs remercient sincèrement Monsieur Stefan Nellen, Président du conseil d'administration de la société TRIDEL SA de son soutien et encouragement précieux qui ont permis la réalisation de cette étude.

\section{Références}

AFTES (Association française des travaux souterrains). 2003. Caractérisation des massifs rocheux utile à l'étude et à la réalisation des ouvrages souterrains. Recommandations de l'association française des tunnels et de l'espace souterrain, GT1R1F1. Tunn Ouvrages Souterr 177.

Hoek \& Brown. 1980. Underground excavations in Rock-Institution of Mining and Metallurgy.

Hoek E, Carranza-Torres CT, Corkum B. 2002. Hoek-Brown failure criterion-Edition, Proceedings of the 5th North American Rock Mechanics Symp., Toronto, Canada.

ISRM. 1983. Suggested methods for determining the strength of rock materials in triaxial compression: Revised version. Int J Rock Mech Min Sci Geomech Abs 20(6): 285-290.

KARAKAS \& FRANÇAIS SA. 2004. Étude géologique du Déchoduc TRIDEL - Rapports établis pour TRIDEL SA.

Sari M. 2010. A Simple approximation to estimate the Hoek-Brown parameter " $\mathrm{m}_{\mathrm{i}}$ " for intact rocks: EUROCK 2010, Rock Mechanics Symp, Lausanne, Switzerland, June 2010.

Citation de l'article : Mustafa Gencer, Jean-François Mathier, Francis Noverraz, Olivier Français. Description des propriétés mécaniques de la Molasse aquitanienne de Lausanne. Rev. Fr. Geotech. 2018, 154, 4. 